\title{
A Method of Recommending Physical Education Network Course Resources Based on Collaborative Filtering Technology
}

\author{
Zhihao Zhang (i) \\ College of Physical Education, Xinyang Normal University, Xinyang Henan 464000, China \\ Correspondence should be addressed to Zhihao Zhang; 2011010115@st.btbu.edu.cn
}

Received 11 August 2021; Revised 17 September 2021; Accepted 19 September 2021; Published 28 October 2021

Academic Editor: Muhammad Usman

Copyright (c) 2021 Zhihao Zhang. This is an open access article distributed under the Creative Commons Attribution License, which permits unrestricted use, distribution, and reproduction in any medium, provided the original work is properly cited.

\begin{abstract}
Through the current research on e-learning, it is found that the present e-learning system applied to the recommendation activities of learning resources has only two search methods: Top-N and keywords. These search methods cannot effectively recommend learning resources to learners. Therefore, the collaborative filtering recommendation technology is applied, in this paper, to the process of personalized recommendation of learning resources. We obtain user content and functional interest and predict the comprehensive interest of web and big data through an infinite deep neural network. Based on the collaborative knowledge graph and the collaborative filtering algorithm, the semantic information of teaching network resources is extracted from the collaborative knowledge graph. According to the principles of the nearest neighbor recommendation, the course attribute value preference matrix (APM) is obtained first. Next, the course-predicted values are sorted in descending order, and the top T courses with the highest predicted values are selected as the final recommended course set for the target learners. Each course has its own online classroom; the teacher will publish online class details ahead of time, and students can purchase online access to the classroom number and password. The experimental results show that the optimal number of clusters $k$ is 9 . Furthermore, for extremely sparse matrices, the collaborative filtering technique method is more suitable for clustering in the transformed lowdimensional space. The average recommendation satisfaction degree of collaborative filtering technology method is approximately $43.6 \%$, which demonstrates high recommendation quality.
\end{abstract}

\section{Introduction}

The Internet era, supported by big data and Internet of things, is a period of rapid development within the domain of knowledge and information curriculum resources. The curriculum resources for sports health and rehabilitation are also more abundant and diverse [1]. Reasonable, scientific, and effective integration and utilization of curriculum resources are bound to be beneficial to the improvement of the construction level of sports health and rehabilitation. Therefore, it is very important to attribute reputation to sport health and rehabilitation and strengthen their integration with curriculum resources [2]. From the perspective of the composition of general curriculum resources, this includes mainly four main parts: (a) teacher team; (b) students; (c) teaching materials; and (d) social resources [3].

With the popularization of the Internet, traditional training schools began turning to the front in 2000, and online learning tools, platforms, and content began emerging in large numbers. "Smart Education" was proposed by Peng et al. in a speech in 2008. Around 2012, online learning ushered in a spurt of growth and professional online learning platforms were born in large numbers, mainly Internet companies (e.g., Ape Guidance, Job Gang, and VIPKID), with diversified content formats. In 2020, due to the impact of the new crown pneumonia epidemic, that is, COVID-19, all universities and education institutes across the country and globe have started online learning management systems [4]. Moreover, employers in various industries also start working from home. Since then, China's online learning began to enter the world stage [5].

Sheshasaayee et al. conducted an in-depth study of the Moodle learning management system in 2017. Moodle not only provides rich learning resources but also has a learning record tracking function, allowing lecturers to freely choose the teaching mode according to the actual situation of the 
learner. At the same time, learners can also choose courses freely to achieve the purpose of personalized teaching [6]. Jones et al. studied the impact of the Knewton-based adaptive learning recommendation engine on learning effects in 2018. The Knewton platform provides powerful functions for learners and classroom-related analysis data to predict their future performance, enabling lecturers to achieve differentiated education based on individuals. Learners obtain personalized curriculum recommendations through systematic analysis and assessment of knowledge and ability, which provides the possibility to realize automated and personalized learning [7]. CIRCSIIVI-Tutor of the University of Illinois in the United States can support personalized services for distance online education and can help to solve learners' problems by establishing an intelligent system based on language dialogs.

This paper proposes a method for recommending physical education network course resources based on collaborative filtering technology. The user-based collaborative filtering method is a way to find out similar neighbors of the target user by calculating the similarity between user behavior and other user behavior (ratings, comments, etc.). Then, the interest or preference of the target user is predicted based on the interest or preference of similar neighbors to provide the user with suggestions. This article introduces an optimized theoretical model, a better collaborative filtering technology for a personalized recommendation of physical education online course resources. The proposed method pays attention to discussing the structure of the model and the implementation of the corresponding scoring mechanism and algorithm and analyzes the three important technologies in the personalized learning resource recommendation model. It is observed that e-learning-related personnel can effectively use collaborative filtering technology in the search process, to improve the efficiency of the personalized recommendation system of learning resources. The principal contributions of our research are highlighted as follows:

We design a novel method for recommending physical education network course resources based on collaborative filtering technology

User-based collaborative filtering is to find out the similar neighbors of the target user by calculating the similarity between user behavior and other user behavior

pay attention to discussing the structure of the model and the implementation of the corresponding scoring mechanism and algorithm and analyze the three important technologies in the personalized learning resource recommendation model

The rest of the paper is organized as follows: in Section 2, we briefly discuss the related literature review in terms of collaborative filtering technology. In Section 3, physical education teaching network course resource recommendations are described. In Section 4, we describe the experimental study and results analysis. Finally, Section 5 illustrates the final thoughts and several directions for future research and investigation.

\section{Collaborative Filtering Technology}

In this section, we offer an overview of the basic terminologies including calculation of content, functional interests, and prediction of the interest degree.

2.1. Content Interest Calculation. The number of views can reflect the user's degree of interest in different data. Assume that the user $u$ interest in Web big data $O$ increases as the number of times that $O$ is viewed by other users increases, and at the same time, it shows an inversely decreasing trend with the frequency of browsing. Then, you can use the TFIDF algorithm, combined with the number of browsing times to obtain the user's interest in big data [8].

TF-IDF is an algorithm for estimating the importance of words to a document in a document collection. It can predict the importance of a target in a dimensional space. Assuming that any user in the recommendation system is regarded as a document, the big data browsed by the user is a certain vocabulary in the document, and the user's preference for the data is obtained according to the number of occurrences of the vocabulary.

The reference assumes that, in the recommendation system, there is a set $u^{\prime}=\left\{u_{1}^{\prime}, u_{2}^{\prime}, \ldots, u_{x}^{\prime}\right\}$ with $\chi$ users and a set $\delta$ with $o^{\prime}=\left\{o_{1}^{\prime}, o_{2}^{\prime}, \ldots, o_{\delta}^{\prime}\right\}$ big data. The number of times the user $u^{\prime}{ }^{\prime}$ views the data $o^{\prime}{ }^{\prime}$ is $R_{i^{\prime}, j^{\prime}}$, and the importance of the web data to the user is calculated by the word frequency $T F_{\chi, \delta}$ :

$$
\mathrm{TF}_{\chi, \delta}=\frac{\mathrm{R}_{i^{\prime}, j^{\prime}}}{\sum_{k \in 0} R_{i^{\prime}, j^{\prime}}} .
$$

Here, $\sum_{k \in 0} R_{i^{\prime}, j^{\prime}}$ represents the sum of the number of times the user browses all the data in the collection [9]. If the number of users who have viewed the data $o^{\prime}{ }_{j^{\prime}}$ is represented as $\chi_{o j}$, the importance of the data $o^{\prime}{ }_{j^{\prime}}$ to all users can be obtained by the reverse document frequency $I D F_{o j}$ :

$$
\mathrm{IDF}_{\mathrm{oj}}=\lg \frac{x}{x_{o j}} .
$$

Therefore, the definition formula of the user's interest in the data content is obtained:

$$
\mathrm{C}_{\chi, \delta}=T F_{\chi, \delta} \times I D F_{o j}=\frac{R_{\chi \delta}}{\sum_{k^{\prime} \in 0} R_{\chi k^{\prime}}} \times \lg \frac{x}{x_{o j}} .
$$

The interest degree values of the user $u_{i^{\prime}}^{\prime}$ for all the data in $o^{\prime}=\left\{o_{1}^{\prime}, o_{2}^{\prime}, \ldots, o_{\delta}^{\prime}\right\}$ constitute $u_{i^{\prime}}^{\prime}$ interest degree vector $C_{x}=\left(C_{x, 1}, C_{x, 2}, \ldots, C_{x, m}\right)$.

2.2. Functional Interest Calculation. There are big differences in the number of users browsing different types of data. The number of views can be increased indefinitely. At the same time, users are not necessarily uninterested in data with zero views, and they may have no experience in using it [10]. To reflect the user's interest more scientifically through the number of views and avoid mass information annihilating the user's interest in other data. This article defines an initial value for the data that the user has not browsed and 
introduces the Sigmoid function to standardize the number of browsing times to obtain the user's interest in the data function.

The Sigmoid function is a common $S$-type function, and its definition is as follows:

$$
S\left(x^{\prime}\right)=\frac{1}{1+e^{-x}}
$$

This function is continuous, smooth, and monotonic and its value range is $(0,1)$, and it is symmetric about the center of $(0,0.5)$, which is a threshold function with better performance. In the interval $-\infty, 0$, the nonlinear growth trend of the block after the slowness appears, and the growth trend of the $0,+\infty$ in the interval is fast first and then slow.

In the recommendation system, the calculation formula for the functional interest ${ }_{F i}{ }^{\prime} j^{\prime}$ of the user $u_{i^{\prime}}^{\prime}$ to the data $o^{\prime}{ }_{j}{ }^{\prime}$ is as follows:

$$
F i^{{ }^{\prime}{ }^{\prime}{ }^{\prime}}=\frac{1}{1+e^{-\left(R_{i}^{\prime} j^{\prime}-R_{i^{\prime}}^{\prime}\right)}} .
$$

The value of formula (5) is in the range of 0,1 , which shows a nonlinear and monotonous increasing trend with the increase of the number of browsing. $R_{i^{\prime}}^{\prime}$ indicates the average number of times the user $u_{i^{\prime}}^{\prime}$ views all the data viewed. This formula more intuitively reflects the user's interest, and in the ${ }_{R i^{\prime} j^{\prime}}=0$ situation, defines the initial value for the functional interest.

From formula (5), it can be concluded that the functional interest degree of the user $u_{i^{\prime}}^{\prime}$ for all the data in $o^{\prime}=\left\{o_{1}^{\prime}, o_{2}^{\prime}, \ldots, o_{\delta}^{\prime}\right\}$ constitutes the functional interest vector

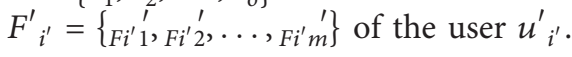

2.3. Prediction of the Comprehensive Value of Interest Degree. After obtaining the user content and functional interest, select the top $\phi$ users with the highest similarity to the target user $u_{a^{\prime}}$ to form the nearest neighbor set $G_{a^{\prime}}$, and use the following formula to predict the interest of the target user $u_{a^{\prime}}^{\prime}$ in the target data $o_{q^{\prime}}^{\prime}$ :

$$
\operatorname{pred}\left(u_{a^{\prime}, o^{\prime}}^{\prime}\right)=\bar{F}_{q^{\prime}}^{\prime}+\frac{\sum_{u_{b^{\prime} \in N^{\prime}}{ }_{a^{\prime}}} S_{a^{\prime} b^{\prime}} \tilde{\mathrm{A}}-\left(\mathrm{F}_{\mathrm{b}^{\prime} \mathrm{a}^{\prime}}-\overline{\mathrm{F}}_{\mathrm{a}^{\prime}}^{\prime}\right)}{\sum_{u_{b^{\prime} \in N^{\prime}{ }_{a^{\prime}}} S_{a^{\prime} b^{\prime}}}} .
$$

Here, $F^{\prime} a^{\prime}$ is the average functional interest of the target user for browsing all the data. After predicting the comprehensive interest of Web big data through the infinite depth neural network, the last $\lambda$ big data items can be collaboratively filtered according to the predicted value.

\section{Physical Education Teaching Network Course Resource Recommendation}

Collaborative filtering is also called social filtering. The userbased collaborative filtering algorithm is mainly divided into two steps:

(1) Find a collection of users with similar interests to the target user.
(2) Find items that are liked by the users in this collection and recommended to the target users that they have not heard of. The user-based collaborative filtering algorithm is only suitable for systems with few users. If there are many users, the cost of calculating the user interest similarity matrix will become very high. The increase in the space complexity and time complexity of the operation and the increase in the number of users are like the square relationship [11]. The recommendation result of this algorithm will not be updated immediately with the user's new behavior, and it is difficult to provide a convincing recommendation explanation to the user.

Based on the collaborative knowledge graph, personalized recommendations are made to network resources. In the network resources, knowledge points are distributed on different web pages. The directed acyclic graph is set as

$$
G=(V, E) \text {. }
$$

Here, $V$ represents the collection of all knowledge in the knowledge graph while $E$ represents the collection of relationships between different knowledge points in $V$, which are mainly divided into two different states, connected and disconnected.

The calculation formula of individual contribution value of network resource knowledge is given as follows:

$$
f(i)= \begin{cases}1 ; & \text { if }(|\operatorname{suc}(\mathrm{i})=0| \operatorname{or}(|\operatorname{pre}(\mathrm{i})=|) 0 \mid) \\ \frac{\operatorname{suc}(i)}{|\operatorname{pre}(\mathrm{i})|}, & \text { others }\end{cases}
$$

At this stage, the centrality calculation formula is a relatively common research method through the degree value of the node, starting from multiple solution angles, and fully reflecting the centrality between each node [12]. Related research results show that the centrality of network resources is the result of the interaction and contribution of the knowledge graph. The specific calculation method of centrality is given as follows:

$$
\mathrm{I}_{\mathrm{i}}=\alpha \delta_{\mathrm{i}}+\gamma \sum_{\mathrm{j}=1} \delta_{\mathrm{j}}+\cdots \gamma^{\mathrm{m}} \sum_{\mathrm{j}=\mathrm{n}} \delta_{\mathrm{j}}
$$

Here, $I_{i}$ represents the centrality of each knowledge point; $\delta$ represents the contribution value of different knowledge points; $\alpha$ represents the evaluation coefficient; and $\gamma$ represents the evaluation coefficient.

For different learning resources, it can be expressed as

$$
I_{i}=\left\{\left(e_{1} w_{1 i}\right),\left(e_{1} w_{2 i}, \ldots,\left(e_{n} w_{n i}\right)\right)\right\}
$$

If $T F$ is set to represent word frequency, the calculation formula for $T F$ is as follows:

$$
\mathrm{TF}_{i j}=\frac{n_{i j}}{\sum_{k} n_{k j}} .
$$

Here, $n_{i j}$ represents the number of times the network resource appears in the text. 
Set $I D F$ to represent the frequency of reverse documents. The main idea is the less the number of documents containing a word, the higher the ability of this word to distinguish between documents. The specific calculation formula is given as follows:

$$
\mathrm{IDF}_{\mathrm{i}}=\log \frac{|D|}{\left|j: e_{i} \in d_{j}\right|+1} .
$$

Here, $|D|$ represents the total number of documents in network resources and $\left|j: e_{i} \in d_{j}\right|$ represents the number of documents contained in the word and adding 1 to the denominator is mainly to prevent the occurrence of 0 [13].

To improve the data processing speed of the entire algorithm, all experimental data need to be initialized and normalized. The following specific calculation formulas are given:

$$
v=\text { uniform }\left(-\frac{6}{\sqrt{d}},-\frac{6}{\sqrt{d}}\right) \text {. }
$$

At the same time, the entity and relationship vectors are normalized and the following calculation formula can be obtained:

$$
v^{\prime}=\frac{v}{\|v\|}
$$

Based on the above analysis, the quantitative representation method of the items in the knowledge graph and the collaborative filtering algorithm are combined, and the semantic information of the network resources is extracted from the collaborative knowledge graph to achieve the personalized recommendation of the network resources [14].

Since the newly registered user in the course selection system has no record of course selection, they cannot directly use the collaborative filtering algorithm to find similar neighbors to recommend personalized course selection for them. Here, we adopt the nearest neighbor recommendation based on the attribute value preference matrix. The major two prerequisites' steps are as follows:

(1) The user has filled in basic information, such as name, student ID, and major, and the learning style measurement scale when registering.

(2) The system automatically generates a student model based on the user's registration information and learning style and converts the model into a vector space model. With this premise, the algorithm flow of the recommendation engine is as follows:

$$
\begin{aligned}
P(u, j[k]) & =\frac{\sum_{h-1}^{H} R_{h}(u, G j([k]))}{H} . \\
\mathrm{APM} & =\left[\begin{array}{cccc}
P(u, 1[1]) & P(u, 2[1]) & \cdots & P(u, t[1]) \\
P(u, 1[2]) & P(u, 2[2]) & \cdots & P(u, t[2]) \\
\cdot & \cdot & \cdots & \cdot \\
P(u, 1[i]) & P(u, 2[i]) & \cdots & P(u, t[i])
\end{array}\right] .
\end{aligned}
$$

$$
\operatorname{sim}(u, v)=\frac{\left\|A_{u, v}\right\|}{1+\sum_{i=1}^{\left\|A_{u, v}\right\|} \sqrt{\left|R_{u, i}-R_{v, i}\right|}}
$$

(1) According to the student model and the course selection record database, generate a student-course selection matrix of order $R(m \times n)$ for each student in the student space (if the student has taken the course, then the score of the course is set to 1 ; otherwise it is 0 ).

(2) Use the cosine similarity formula (14) to calculate the similarity between the target learner $u$ and any learner $\mathrm{v}$ in the student space. By calculating the similarity, the first several learners with the largest similarity are taken as the initial neighbor set IniSet $(u)$ of the standard learner $u$. Determine the size of IniSet $(u)$ according to the number of learners in the student space and the corresponding ratio. At the same time, it is necessary to ensure $k \ll n$, which can reduce the time complexity of the algorithm, thereby improving the real-time performance of the algorithm.

(3) Generate a new student-course selection matrix $R^{\prime}$ based on the initial neighbor set of the target learner and the target learner $u$ generated in the second step.

(4) According to the course selection record database, map the courses taken by the target learner from the course information table to the course attribute value table [15]. Because different courses have different attributes, each attribute has a different score for each course, so we should first count the score set $G(j[k])$ of each attribute value. $G(j[k])$ represents the $k$-th value of the course attribute $j$ (the number of courses determines the value of $k)$, and $P(u, j[k])$ is used to represent the learner $u$ 's interest preference for the attribute value $G(j[k])$, as shown in the following formula:

Here, $R_{h}(u, G(j[k]))$ represents the score of the learner $u$ on the course attribute, and $H$ refers to the total number of times the learner $u$ has scored the value $G(j[k])$ of the attribute $j$. Using the learner's interest preference $P(u, j[k])$, the learner $u$ 's preference matrix APM for course attribute values can be obtained, as shown in the following formula:

The attributes of the courses in the course selection system will be automatically converted into the corresponding course model after uploading the course, and the course attributes will be stored in the course database. The course information table is shown in Table 1.

(5) Based on the attribute value preference matrix APM generated in the fourth step, the similarity formula is used to calculate the similarity between the target learner $u$ and other students in the student space. The similarity formula is as follows: 
TABLE 1: Course information table.

\begin{tabular}{lllllll}
\hline & Attri (1) & Attri (2) & $C_{1}$ & Attri $(\mathrm{j})$ & $\mathrm{C} 1$ & Attri (i) \\
\hline$C_{1}$ & $G_{1,1}$ & $G_{1,2}$ & $\ldots$ & $G_{1, j}$ & $\ldots$ & $G_{1, \mathrm{t}}$ \\
$C_{2}$ & $G_{2,1}$ & $G_{2,2}$ & $\ldots$ & $G_{2, j}$ & $\ldots$ & $G_{2, t}$ \\
$\ldots$ & $\ldots$ & $\ldots$ & $\ldots$ & $\ldots$ & $\ldots$ & $\ldots$ \\
$C_{i}$ & $G_{i, 1}$ & $G_{2,1}$ & $\ldots$ & $G_{i, j}$ & $\ldots$ & $G_{\mathrm{i}, \mathrm{t}}$ \\
\hline
\end{tabular}

Here, $\left\|A_{u, v}\right\|$ represents the size of the collective score attribute value set of learner $u$ and learner $v$; learner $u$ 's score for the $i$-th attribute is expressed as $R_{u, i}$; and the value should be less than $\left\|A_{u, v}\right\|$.

(6) Sort the similarity between the target learner and other learners in descending order. Intercept the first $k$ learners with the largest similarity as the nearest neighbors of the target learner; store them in the nearest neighbor set $U\left(U=\left\{u_{1}, u_{2}, \ldots, u_{k}\right\}\right)$ of the target learner, and the $\underline{\mathrm{U}}$ set does not contain $u$.

(7) Find out which courses they have taken from the set $U$ of the nearest neighbors of the target learners and store them in the set $I U$. Then, delete the courses that the target learner has taken from the set $I U$, and the remaining courses can be used as the recommended set of elective courses for the target learner $\mathrm{C}_{\mathrm{can}}$.

(8) Suppose the number of items in $C_{c a n}$ is $Q$, and the number of recommended courses is $\mathrm{T}$; then, when $Q \leq T, C_{c a n}$ is directly used as the recommended set $C_{\text {rec }}$; when $Q>T$, first use the prediction formula (17) to calculate the target learner's prediction value for the courses in the recommendation set $C_{c a n}$. Then, the predicted value is sorted in descending order, and the top $T$ courses with the highest predicted value are selected as the final recommended course set $C_{\text {rec }}$ for the target learner.

\section{Experimental Study}

4.1. Results and Analysis of Data Experiments on the Resources of the Network Courses of Physical Education. A data set of physical education online course resources on a certain platform is used here, and users with less than 20 clicks are deleted based on the data set to form 1,486 users' browsing information on 1,430 pages. Randomly extract 1,250 records from this data set and divide them into 1000 training sets and 50 test sets (divided into 5 groups of current users). To test the prediction quality under different amounts of information, each group of visible user interests is randomly selected. The number of visible interest pages of the user is from 5 to 20 pages, named Given5 $\sim$ Given 20 , respectively. The sparsity level $\psi_{\mathrm{gl}}$ of this data set is as follows:

$$
\psi_{g l}=1-50561 / 1250 \times 1430=0.9717 .
$$

It can be observed that this data set is sparser than the MovienLens data set $\left(\psi_{m l}=0.9369\right)$ and belongs to the severe sparse level. The test environment and system's characteristics are shown in Table 2.
4.2. Evaluation Index. To further evaluate the effect and accuracy of the recommendation, the recommendation system can also use three commonly used evaluation indicators in the field of information retrieval as the standard to measure the recommendation effect, namely, Precision, Recall and F1 value. The data set is divided into a training set and a test set. The model of the recommendation algorithm is learned and parameter adjusted on the training set; then, the recommendation result is calculated on the test set to obtain the evaluation result:

(1) Precision: the proportion of recommended pages (hit) that are correctly identified in the Top- $\mathrm{N}$ recommendation set:

$$
\text { precision }=\frac{\mid \text { test } \cap \text { TopN } \mid}{\mathrm{N}} .
$$

(2) Recall rate (recall): the proportion of recommended pages (hit) that are correctly identified in all test sets:

$$
\text { recall }=\frac{\mid \text { test } \cap \text { TopN } \mid}{\mid \text { test } \mid} .
$$

(3) $F_{1}$-measure: an indicator that integrates the above two evaluation indicators. Considering that the accuracy and recall rates of different systems are mutually high and low in some cases, it is impossible to make a judgment using only the above two indicators. Therefore, the $F_{1}$ value is introduced, and these two indicators can be used at the same time to fully explain the performance of the algorithm. The calculation formula of $F_{1}$ is as follows:

$$
F_{1}=\frac{2 \times(\text { precision } \times \text { recall })}{\text { precision }+ \text { recall }} \text {. }
$$

4.3. Experimental Results and Discussion. According to the steps of the new algorithm, the training model $m$ is first smoothed, the pages with no interest value are preliminarily predicted through the item-based collaborative filtering algorithm, and the filled training model $m$ ' is obtained. After the principal component analysis of $m^{\prime}$, its cumulative contribution rate is shown in Figure 1. The solid line part in Figure 1 represents the cumulative contribution rate of each principal component of the smoothed model $m$ '. The first 58 and the first 147 principal components with a cumulative contribution rate of $80 \%$ and $90 \%$ are selected as the dimensions of the transformed vector, respectively. Clustering is performed in a low-dimensional space. Table 3 describes the naming of the contrast algorithm used in this experiment:

$$
\text { MAE }=\frac{\sum_{i=1}^{n}\left|P_{i}-R_{i}\right|}{N} .
$$

Note that the MAE metric, as given by formula (22), represents the average absolute error value index, where $P_{i}$ represents the data predicted value obtained through the 
TABLE 2: Test environment and system's characteristics.

\begin{tabular}{lc}
\hline Processor & Intel(R) Core (TM) i5-3210M CPU @ 250 Hz 250 Hz \\
\hline Memory capacity & $4.00 \mathrm{~GB}$ \\
Operating system & Windows 7 Home Basic 64 bits \\
Hard drive capacity & $5400 \mathrm{rpm}$ \\
Graphics card & NVIDIA GeForce GT 630M + Intel GMA HD 4000 \\
\hline
\end{tabular}

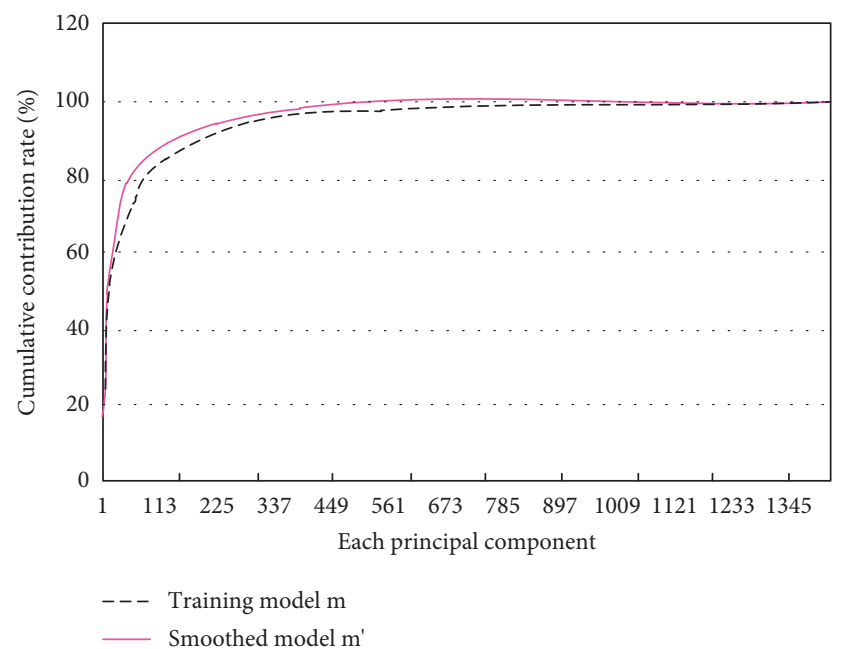

FIgURE 1: The curve comparison of the cumulative contribution rate of the principal components of $m$ and $m$ ' in the log data set.

TABLE 3: Name description of each algorithm.

\begin{tabular}{lc}
\hline Algorithm naming & Algorithm description \\
\hline UPCC & User-based CF \\
KCLUST-CF & K-means clustering \\
PCA90-UPCC & PCA + UPCC \\
PCA-KM90 & PCA + K-means clustering \\
PCA-SOM90 & PCA + SOM clustering \\
\hline
\end{tabular}

algorithm, $R_{i}$ represents the true value obtained from the real data, and $N$ represents the number of samples in the predicted data.

Figure 2 records the influence of the number of clusters on the MAE under the experimental conditions of Given 10. It is preliminarily judged that the optimal number of clusters $k$ should be between 8 and 12 . We might set $k=9$ in future experiments.

To improve the accuracy of the two-stage prediction algorithm proposed in this paper, we designed two sets of experiments. The first set of experiments used different clustering algorithms (K-means clustering and collaborative filtering technology methods) to compare the prediction accuracy. It also compares the classic collaborative filtering algorithm UPCC (user-based collaborative filtering), and the collaborative filtering algorithm based on user clustering. The second set of experiments compared the effect of taking $90 \%$ and $80 \%$ of the cumulative contribution rate of principal components on the quality of prediction. In the experiment, K-means clustering uses Cosine distance

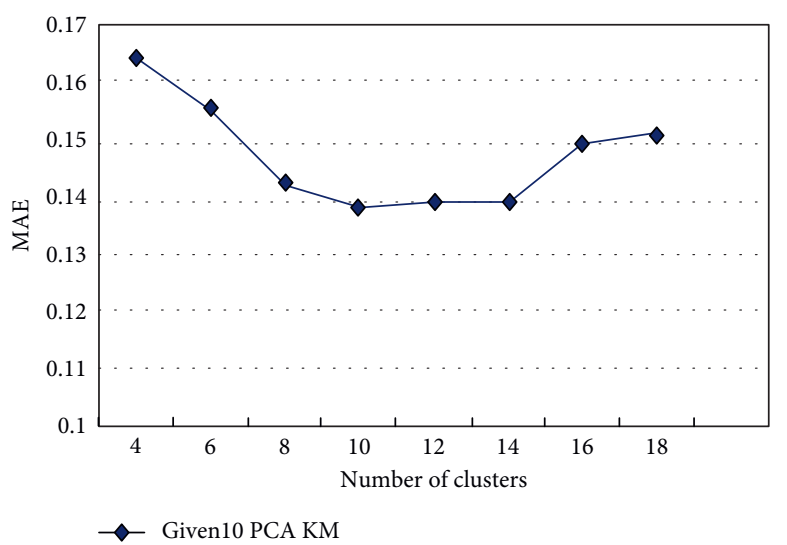

Figure 2: The impact of different cluster numbers on recommendation accuracy.

measurement, and the output of the collaborative filtering technique method is a $3 \times 3$ two-dimensional neuron node, the training step is 1000 , and the learning rate is an exponential decay function.

The experimental results of Figure 3 show that the overall quality of prediction of the algorithm gets more accurate with an increase in the amount of information about historical interest. For extreme sparse matrices, it can be observed that collaborative filtering is more feasible for clustering within the transformed low-dimensional spaces. Moreover, compared with the collaborative filtering algorithm, which is based on user clustering (KCLUST_CF), the traditional UPCC nearest neighbor algorithm still has much better prediction accuracy.

Table 4 summarizes the offline and online computing time complexity of several comparison algorithms. It can be observed that the new algorithm based on effective dimensionality reduction and clustering proposed in this paper has a better real-time recommendation effect.

Several sets of comparative experiments will be conducted on the accuracy of the algorithm to generate recommendations. First, hide $m$ page preferences for each user in the test set. The rest is used as historical preference information to predict the interest value of unknown pages, and on this basis, $\mathrm{N}$ pages with the highest predicted interest value are selected and recommended to the user. If the recommended page appears on a previously hidden page, it is called a hit. In this experiment, $m=10$ and $N=5$, that is, the recommendation model selects the first 5 pages with the highest predicted interest value as the recommendation output to the user. It can be seen from the comparison curve, in Figure 4, that, as the number of $\mathrm{N}$ increases, the accuracy of the recommendation will continue to decline, and finally 


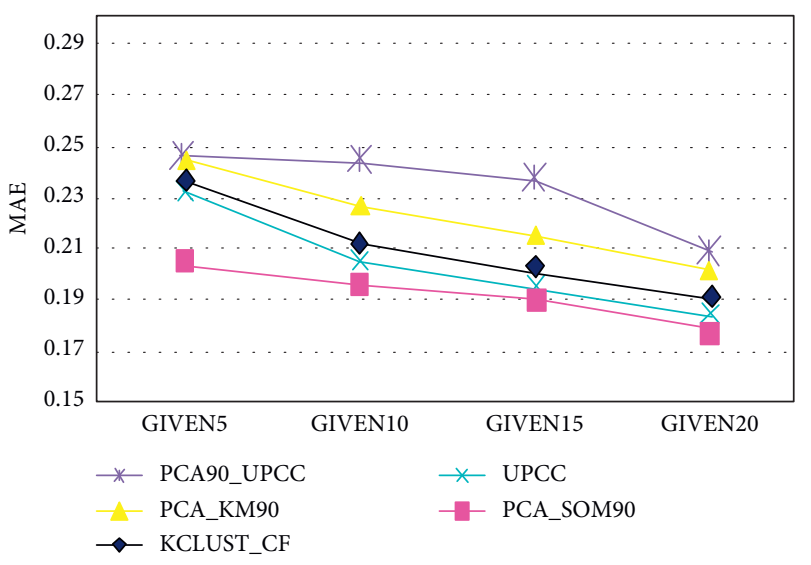

Figure 3: Comparison of prediction accuracy of different algorithms.

TABLE 4: Comparison of time complexity of improved collaborative algorithms.

\begin{tabular}{lcc}
\hline Algorithm & Offline time complexity & Online time complexity \\
\hline UPCC & - & $o(m n)$ \\
KCLUST_CF & $o(m t k)$ & $o(n)$ \\
PCA90_UPCC & $o\left(n^{2} m\right)+o\left(n^{3}\right)$ & $o(m d)$ \\
PCA90_KM & $o\left(n^{2} m\right)+o\left(n^{3}\right)+o(m t k)$ & $o(d)$ \\
PCA90_SOM & $o\left(n^{2} m\right)+o\left(n^{3}\right)+o\left(t^{2} d\right)$ & $o(d)$ \\
\hline
\end{tabular}

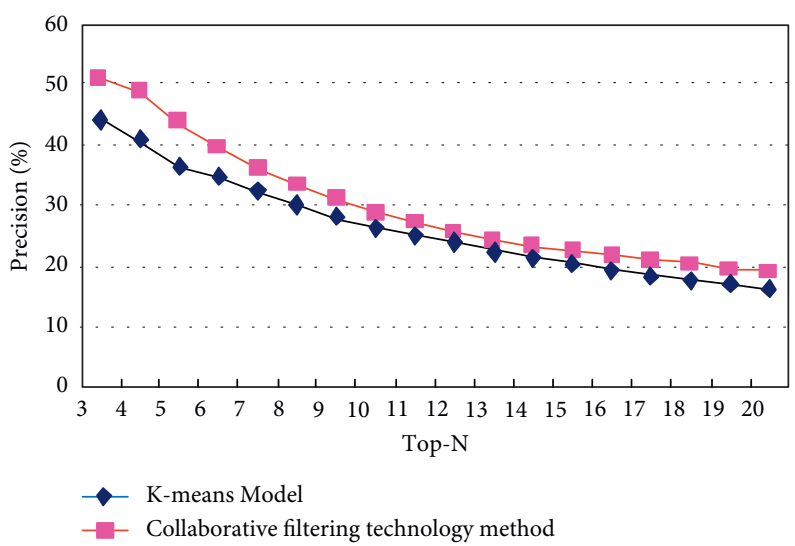

Figure 4: Comparison of recommended accuracy rates.

TABLE 5: Comparison of evaluation indicators of the two models.

\begin{tabular}{lcc}
\hline Metrics & PCA_K-means model & Collaborative filtering technology method \\
\hline Mean precision (\%) & 36.4 & 43.6 \\
Mean recall (\%) & 15.19 & 15.40 \\
Mean F1 & 0.2144 & 0.2277 \\
\hline
\end{tabular}

gradually stabilize. In general, the recommendation performance of the collaborative filtering technology method is better than that of the K-means model. As shown in Table 5, the average recommendation satisfaction degree of the collaborative filtering technology method is approximately $43.6 \%$, while the evaluation recommendation satisfaction degree of the K-means model is somehow $36.4 \%$, which is far less than that of the former method. Therefore, compared with the accuracy rate, the recall rates of the two models are relatively low. The reason that we believe is that the $N$ value and the size of the test set affect the recall rate.

Table 6 reflects the comparison of the statistical prediction accuracy of the two models, as well as the prediction of the degree of interest of the portal page. The prediction accuracy of the collaborative filtering technology method is more accurate than that of the K-means model. This finding 
TABLE 6: Comparison of the recommended quality of the two models (MAE values).

\begin{tabular}{|c|c|c|c|c|}
\hline Model & Given5 & Given 10 & Given 15 & Given20 \\
\hline PCA_K-means & 0.24565 & 0.22685 & 0.2158 & 0.2023 \\
\hline Collaborative filtering technology method & 0.2045 & 0.19705 & 0.1903 & 0.1787 \\
\hline
\end{tabular}

was observed since the average MAE of the proposed method is reduced by approximately 0.03 . Particularly, when there is less predictive historical information (e.g., Given5), the collaborative filtering technology method performs better in predicting quality, and the MAE is reduced by 0.041. Table 6 shows that the larger the historical importation, the lesser the MAE value.

In short, the page recommendation experiment in this data set illustrates that the recommendation method based on collaborative filtering technology shows better prediction accuracy and improves the accuracy of the recommendation. Moreover, the time complexity of the online model is significantly reduced, which is a sign of a more practical online recommendation model.

\section{Conclusions and Future Work}

The fulfillment of individualized educational resource recommendations has become the key problem that has to be solved in intelligent education due to the dual pressures of work and study. Since the personalized recommendation system has become the focus of current research on how to integrate with online teaching as a critical technology to alleviate the problem of cognitive overload or confusion during online learning, student's interests are not static and the recommended results should be updated continuously with the changes of students' interests, which helps to cultivate the potential interests of users. Therefore, the requirements for the system to respond immediately to changes in user interest are very high. To solve the real-time problem of the recommendation system, many scholars have proposed a model-based collaborative filtering algorithm based on user clustering, which has achieved certain results.

Based on the collaborative knowledge graph, this paper combines the collaborative filtering algorithm to extract the semantic information of teaching network resources from the collaborative knowledge graph. According to the nearest neighbor recommendation principle, the course attribute value preference matrix APM is obtained, the course predicted values are sorted in descending order, and the top $T$ courses with the highest predicted value are selected as the final recommended course set for target learners. Finally, the rehearsal platform was put through its paces in terms of functionality and performance, yielding the expected results. The rehearsal platform provides students with appropriate IT learning resources as well as a platform for learning exercises, and the recommendation system not only helps users locate high-quality content that interests them fast but also saves them time and money. The search range of the nearest neighbor is narrowed, the feature information is more concentrated, and a better recommendation effect is obtained in the test on the standard data set and the real data.

\section{Data Availability}

The data used to support the findings of this study are available from the corresponding author upon request.

\section{Conflicts of Interest}

The author declares no conflicts of interest.

\section{References}

[1] C. D. Campbell, B. Challen, K. L. Turner, and M. I. Stewart, "\#DryLabs20: a new global collaborative network to consider and address the challenges of laboratory teaching with the challenges of COVID-19," Journal of Chemical Education, vol. 97, no. 9, pp. 3023-3027, 2020.

[2] D. Darici, C. Reissner, J. Brockhaus, and M. Missler, "Implementation of a fully digital histology course in the anatomical teaching curriculum during COVID-19 pandemic," Annals of Anatomy - Anatomischer Anzeiger, vol. 236, no. 1, Article ID 151718, 2021.

[3] H. Zhu, "Research on Human Resource Recommendation Algorithm Based on Machine Learning," Scientific Programming, vol. 2021, pp. 1-10, 2021.

[4] A. Ranauta, A. R. Tappuni, and P. Coulthard, "HIV Teaching: a dental curriculum which fosters knowledge and attitude," Oral Diseases, vol. 26, no. S1, pp. 123-126, 2020.

[5] A. Huang-Saad, J. Stegemann, and L. Shea, "Developing a model for integrating professional practice and evidencebased teaching practices into BME curriculum," Annals of Biomedical Engineering, vol. 48, no. 2, pp. 881-892, 2020.

[6] A. Sheshasaayee and M. N. Bee, "Evaluating user experience in moodle learning management systems," in Proceedings of 2017 International Conference on Innovative Mechanisms for Industry Applications (ICIMIA), pp. 735-738, IEEE, Bengaluru, India, February 2017.

[7] A. Jones and I. Bomash, "Validating mastery learning: assessing the impact of adaptive learning objective mastery in Knewton alta," in Proceedings of the International Conference on Artificial Intelligence in Education, pp. 433-437, June 2018.

[8] D. Butko, "Over a decade of decibels-Celebrating teaching architectural acoustics within an architecture curriculum to students with various majors and minors," Journal of the Acoustical Society of America, vol. 148, no. 4, p. 2526, 2020.

[9] X. Zhang, "Planning the Structure of University Teaching Staff Based on Multiobjective Optimization Method," Scientific Programming, vol. 2021, pp. 1-10, 2021.

[10] M. Li, C. Bangeranye, M. Frankfurt, and B. Catherine, "Teaching and assessing principles of pharmacokinetics and pharmacodynamics in an integrated medical school curriculum," The FASEB Journal, vol. 34, no. S1, p. 1, 2020.

[11] A. S. V. K. Mohindra, J. G. H. Jie, L. Y. Lim et al., "A student and staff collaborative audit exploring the food and drinks available from a dental teaching hospital outlet," British Dental Journal, vol. 230, no. 1, pp. 32-38, 2021. 
[12] M. S. Brubacher, Z. Lauer, L. M. Eck, and B. Lowry, "Teaching root cause analysis through a collaborative case-based method," Medical Education, vol. 53, no. 11, pp. 1135-1136, 2019.

[13] J. D. Pasternak, A. H. Imisairi, I. H. M. B. Rinkes, and S. Kruijff, "The IAES-supported Malaysian breast and endocrine surgery course: 7 Years of successful collaborative teaching," World Journal of Surgery, vol. 44, no. 8, pp. 2667-2668, 2020.

[14] M. Taj, B. Lalani, N. Madhani et al., "Oncology nursing training: a blended teaching approach in resource-limited countries," Journal of Clinical Oncology, vol. 38, no. 15, Article ID e19189, 2020.

[15] C. A. Green, K. M. Mahuron, H. W. Harris, and P. S O'Sullivan, "Integrating robotic technology into resident training: challenges and recommendations from the front lines," Academic Medicine: Journal of the Association of American Medical Colleges, vol. 94, no. 10, pp. 1532-1538, 2019. 